Design and Development of a New Pyrromethene Dye with Improved Photostability and Lasing Efficiency: Theoretical Rationalization of Photophysical and

\title{
Photochemical Properties
}

Soumyaditya Mula, ${ }^{1}$ Alok K. Ray, ${ }^{2}$ Manas Banerjee, ${ }^{3}$ Tandrima Chaudhuri, ${ }^{3}$ Kamalesh Dasgupta ${ }^{2}$ and Subrata Chattopadhyay ${ }^{1 *}$

${ }^{1}$ Bio-Organic Division, ${ }^{2}$ Laser \& Plasma Technology Division, Bhabha Atomic Research Centre, Mumbai -400085, India. ${ }^{3}$ Chemistry Department, Burdwan University, Burdwan - 713104, India

\begin{tabular}{|l|l|l|l|}
\hline content & page no. & Content & page no. \\
\hline Synthesis & S3 & Tables S1 \& S2 & S9 \\
\hline Laser set-up & S4 & ${ }^{1}$ H NMR of 6 & S10 \\
\hline Cyclic voltammogram of 1 & S5 & ${ }^{13}$ C NMR of 6 & S11 \\
\hline Cyclic voltammogram of 4 & S6 & ${ }^{1}$ H NMR of 7 & S12 \\
\hline $\begin{array}{l}\text { HPLC profile of the } \\
\text { photodegradated products of } \\
\text { the dye 1 }\end{array}$ & S7 & ${ }^{13}$ C NMR of 7 & S13 \\
\hline $\begin{array}{l}\text { HPLC profile of the } \\
\text { photodegradated products of } \\
\text { the dye 4 }\end{array}$ & & ${ }^{1}$ H NMR of 8 & S14 \\
\hline
\end{tabular}




\begin{tabular}{|l|l|l|l|}
\hline${ }^{13}$ C NMR of $\mathbf{8}$ & S15 & Computational Methods & S22 \\
\hline${ }^{1}$ H NMR of $\mathbf{2}$ & S16 & Computational Data of $\mathbf{1}$ & S23-S26 \\
\hline${ }^{13}$ C NMR of $\mathbf{2}$ & S17 & Computational Data of $\mathbf{2}$ & S27-S31 \\
\hline${ }^{1}$ H NMR of 3 & S18 NMR of 3 & Computational Data of $\mathbf{3}$ & S32-S36 \\
\hline${ }^{13}$ H NMR of $\mathbf{4}$ & S19 & Computational Data of $\mathbf{4}$ & S37-S41 \\
\hline${ }^{13}$ C NMR of $\mathbf{4}$ & S20 & Computational Data of $\mathbf{1 1}$ & S42-S45 \\
\hline
\end{tabular}


General Methods. Laser grade Pyrromethene 567 (from Exciton) and Rhodamine 6G (from Lambda Physik) was used without any further purification. The purity of the dye was found to be $>99 \%$, as determined with spectroscopic and chromatographic methods. All the chemicals and spectroscopic grade solvents were purchased from Aldrich, Merck or Sigma and were used without any further purification. The IR spectra were recorded with a Nicolet 430 FT-IR spectrophotometer. The ${ }^{1} \mathrm{H}$ NMR spectra were recorded with a Bruker $200 \mathrm{FT}$-spectrometer in $\mathrm{CDCl}_{3}$ if not mentioned otherwise. The mass spectra (70 eV) were recorded with a MD-80 Fission instrument. The spectrophotometry was carried out at $25{ }^{\circ} \mathrm{C}$ with a Jasco V-550 UV-vis spectrophotometer and Jasco FP 6500 spectrofluorometer using quartz cells of $1 \mathrm{~cm}$ path length. 
Figure S1. Schematic diagram of the Nd-YAG laser pumped broad band dye laser set up, used for comparing the laser efficiencies of the dyes. Different blocks represent 1 - Nd-YAG laser, 2 - second harmonic Generator, 3 - half-wave plate, $\mathbf{4}$ - polarizer plate, 5 - Beam dump, 6 beam splitter (50\%), 7 - power meter, $\mathbf{8}$ - mirror, $\mathbf{9}$ - cylindrical lens, $\mathbf{1 0}$ - dye cell, $\mathbf{1 1}$ - high reflectivity mirror, 12 - output coupler, 13 - power meter.

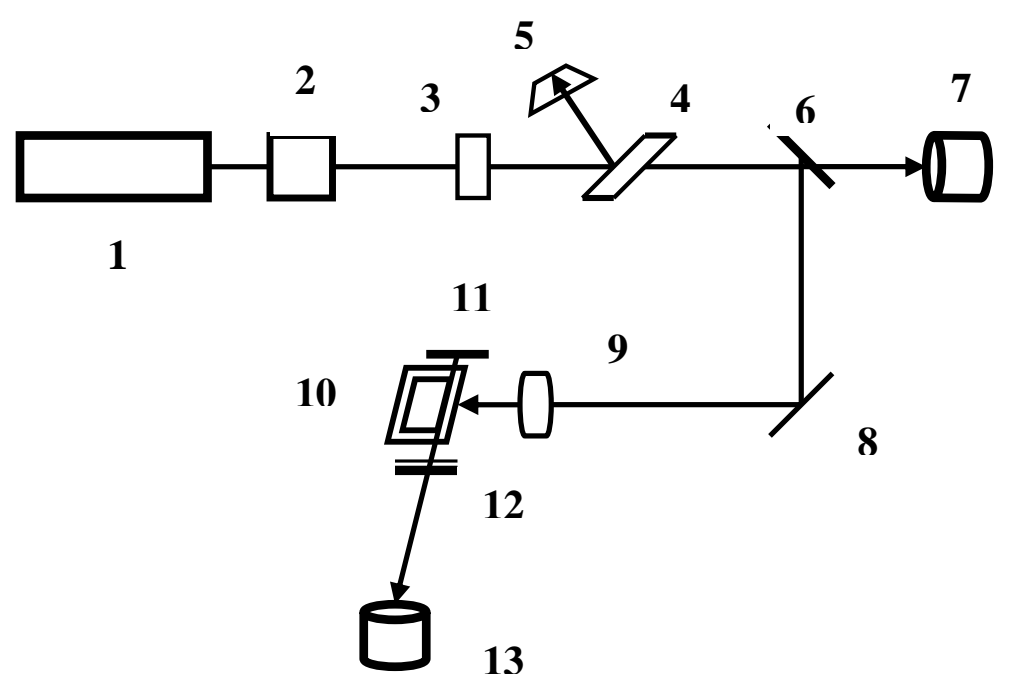


Figure S2. Cyclic voltammogram of dye $\mathbf{1}$ in ethanol.

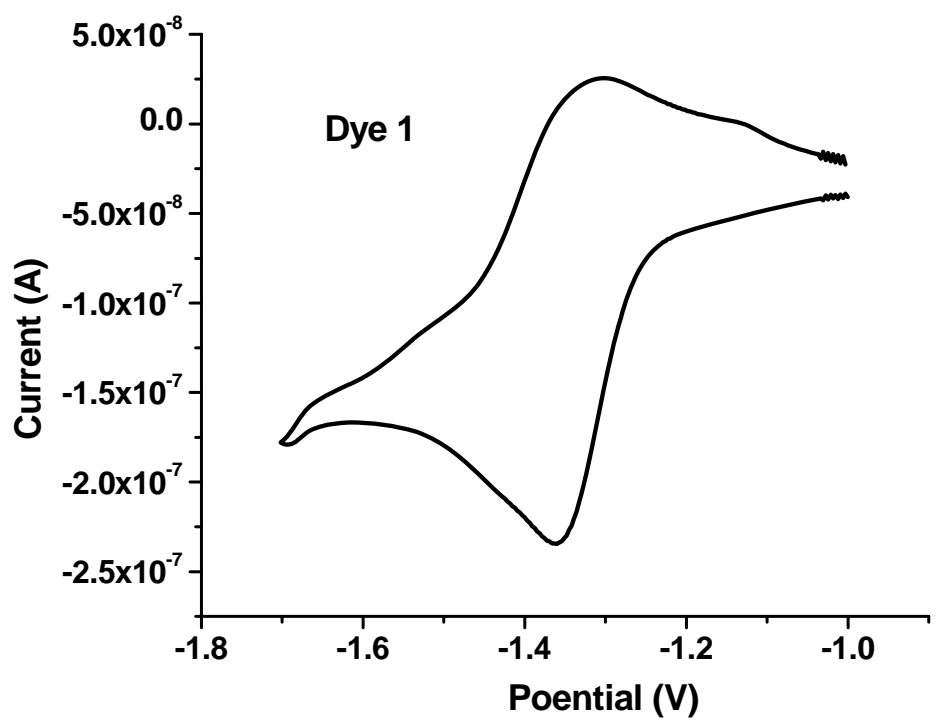


Figure S 3. Cyclic voltammogram of dye $\mathbf{4}$ in ethanol.

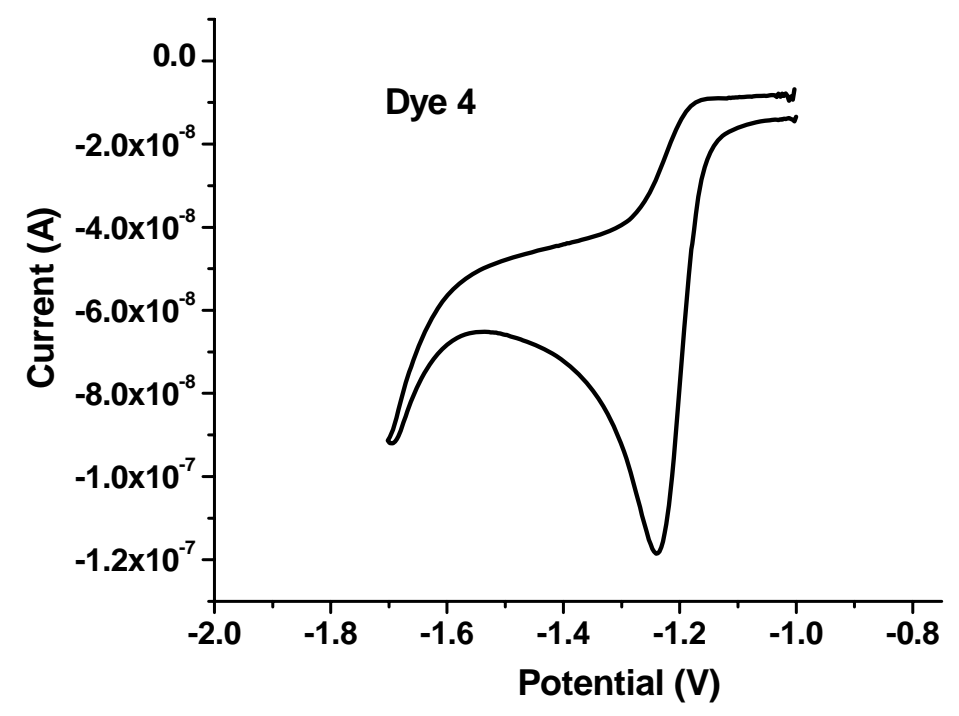


Figure S4. HPLC profile of the photodegradated products of the dye $\mathbf{1 .}$

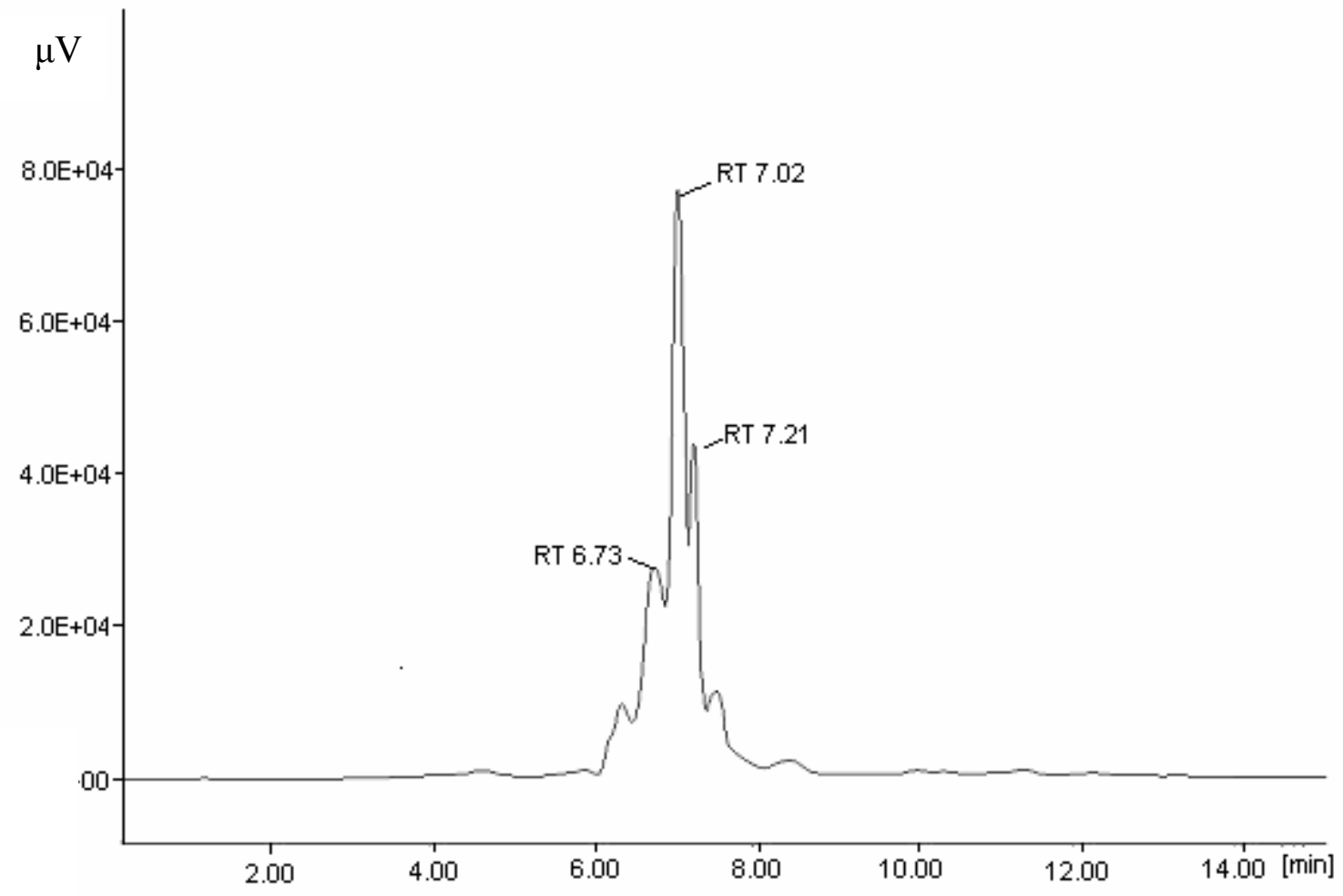


Figure S5. HPLC profile of the photodegradated products of the dye 4 .

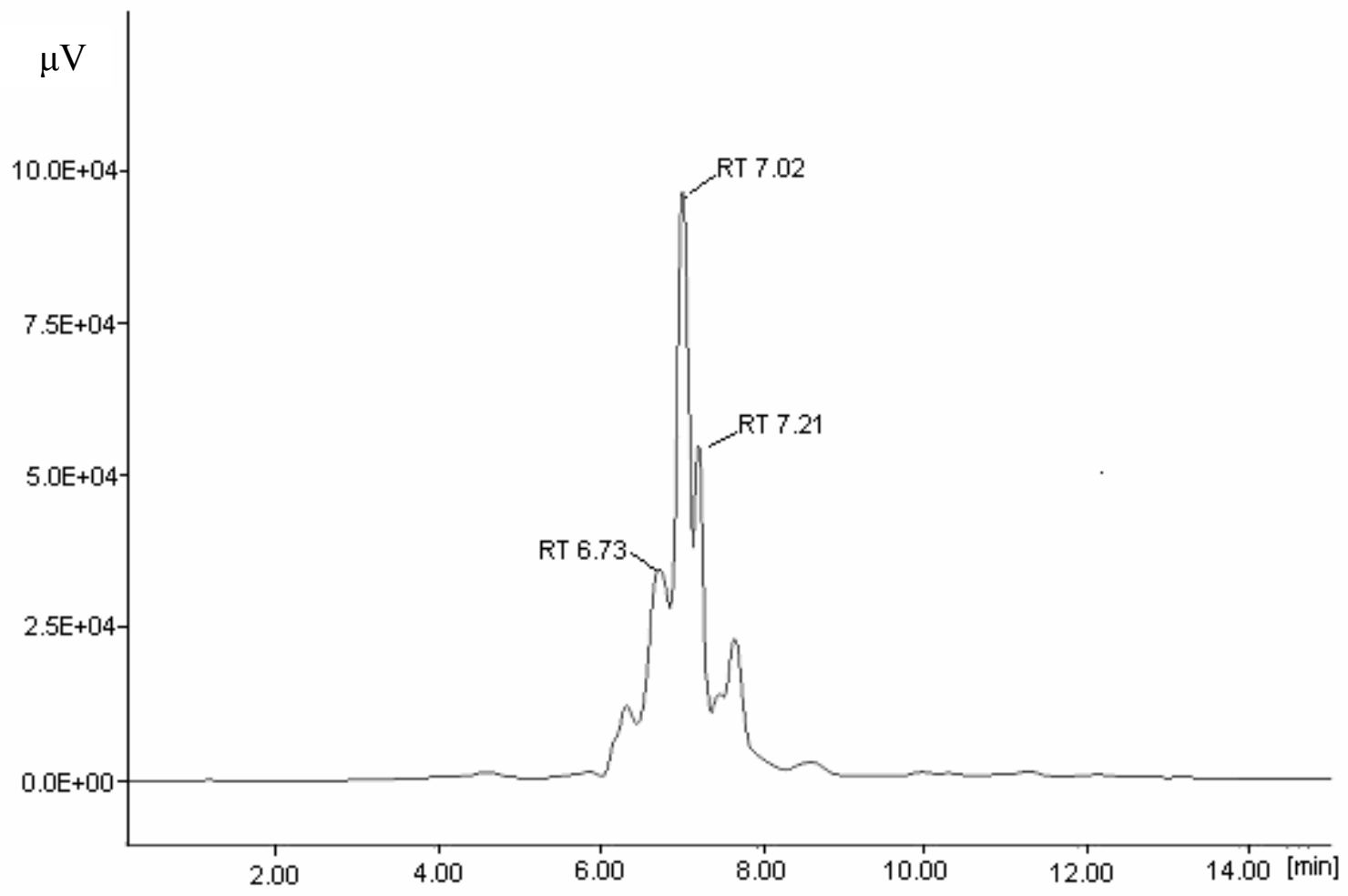


Table S1. Quantification of photodecomposition of 1-4 due to laser irradiation ${ }^{\mathrm{a}}$

\begin{tabular}{ll}
\hline Dye & \% Photodecomposition \\
\hline $\mathbf{1}$ & 18.2 \\
$\mathbf{2}$ & 5.5 \\
$\mathbf{3}$ & 6.1 \\
$\mathbf{4}$ & 7.9
\end{tabular}

\begin{abstract}
${ }^{\mathrm{a}}$ The individual dyes were irradiated under non-lasing conditions by the $532 \mathrm{~nm}$ output of a pulsed Nd:YAG laser for $20 \mathrm{~min}$. The extents of degradation were quantified from the integrated area of the respective HPTLC chromatogram $\left[60 \mathrm{~F}_{254}\right.$ silica gel plate, $10 \% \mathrm{EtOAc/hexane}$, detection at $254 \mathrm{~nm}$ ] using a standard graph prepared separately with the individual dyes. The values are mean of three different experiments.
\end{abstract}

Table S2. Configuration coefficients of $\mathbf{1 - 4}$ for the TDDFT excited singlet ( $\mathrm{S}_{1}$ state)

\begin{tabular}{lllll}
\hline Parameter & Dye 1 & Dye 2 & Dye 3 & Dye 4 \\
\hline HOMO $\rightarrow$ LUMO & 0.4744 & 0.4417 & 0.4425 & 0.4562 \\
HOMO-1 $\rightarrow$ LUMO & 0.4082 & 0.4521 & 0.4508 & 0.3585 \\
HOMO-3 $\rightarrow$ LUMO & - & - & - & 0.2355 \\
\hline
\end{tabular}




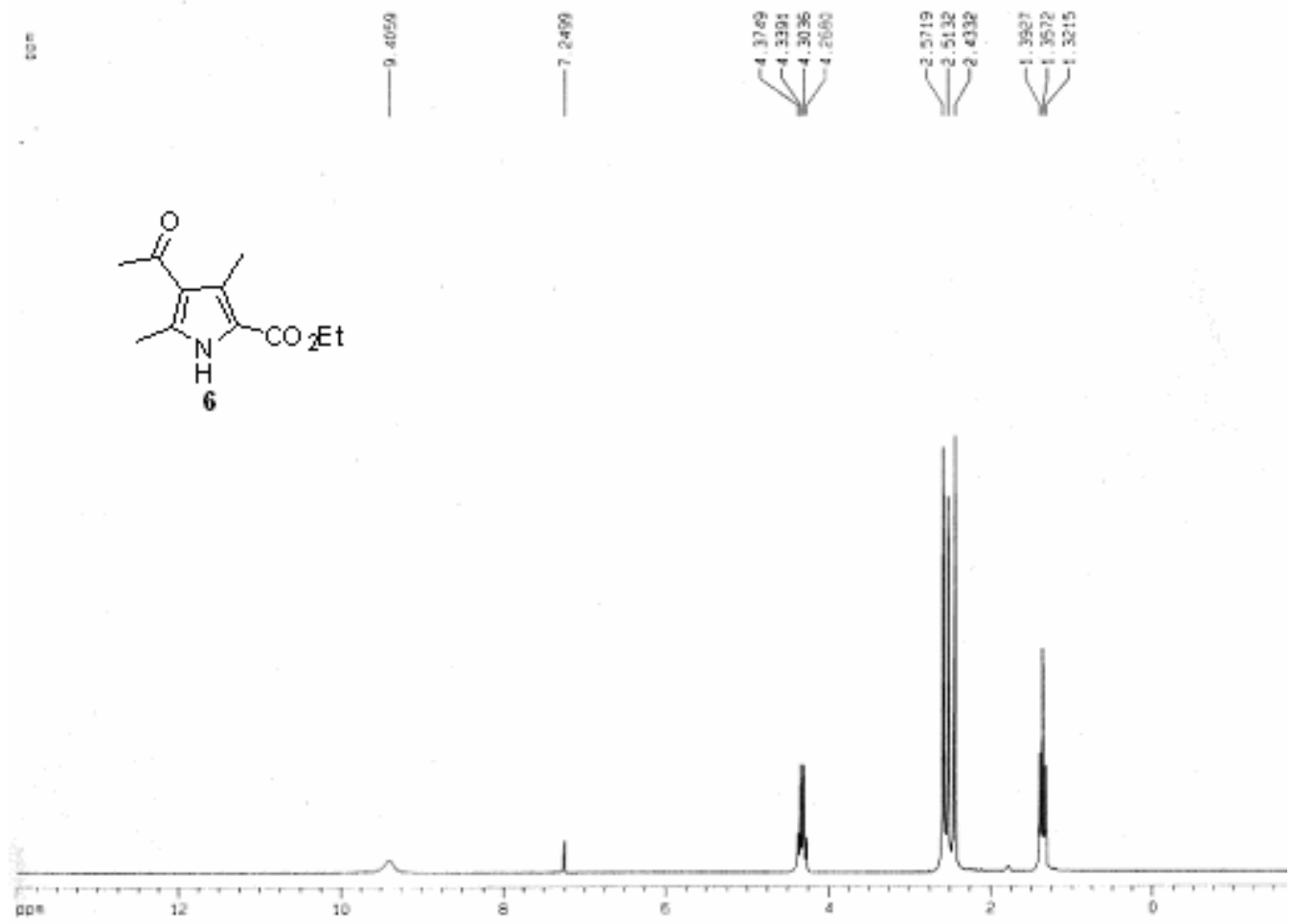




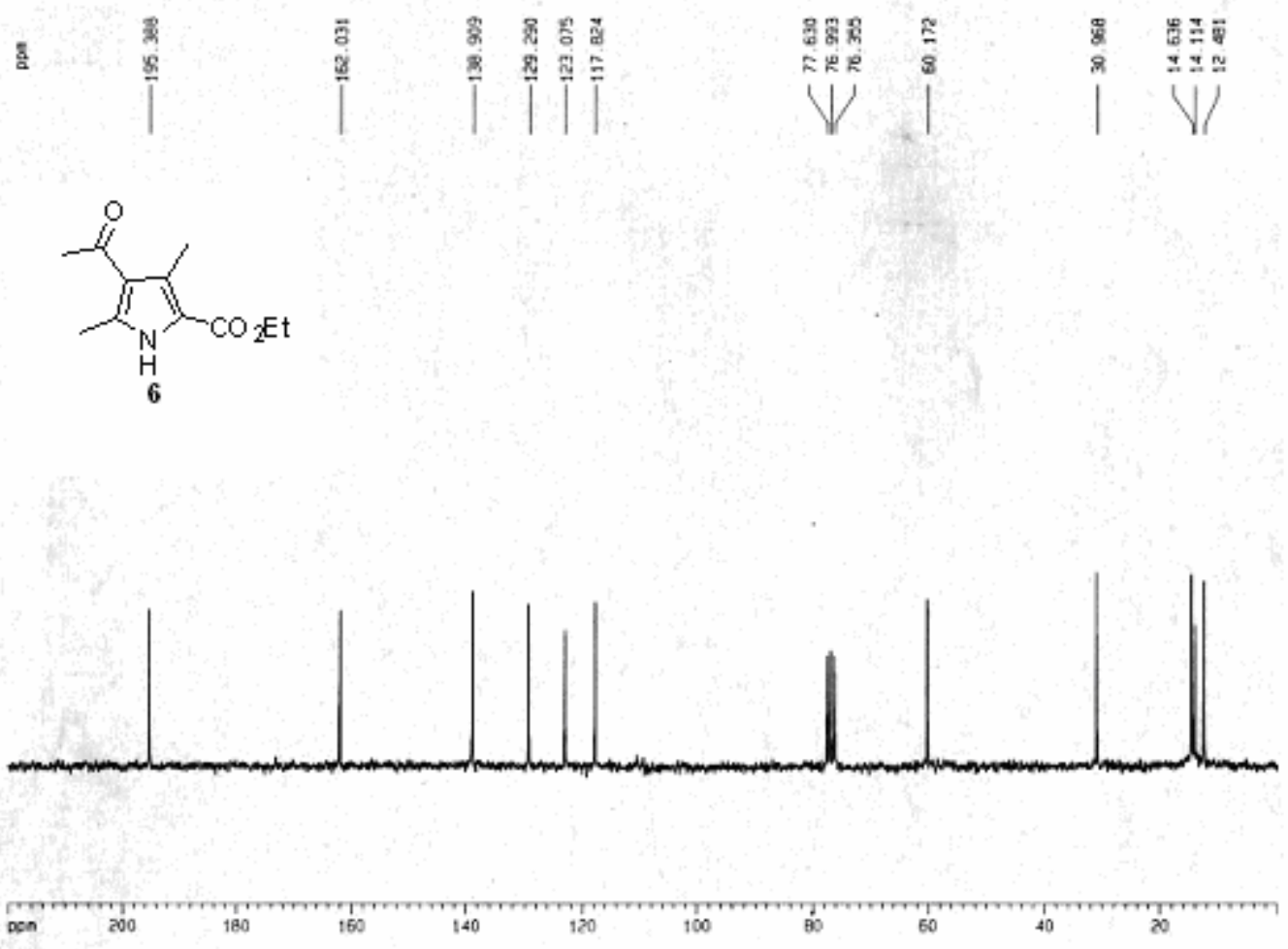


S12

ลิ

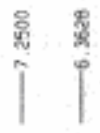

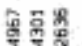

ij

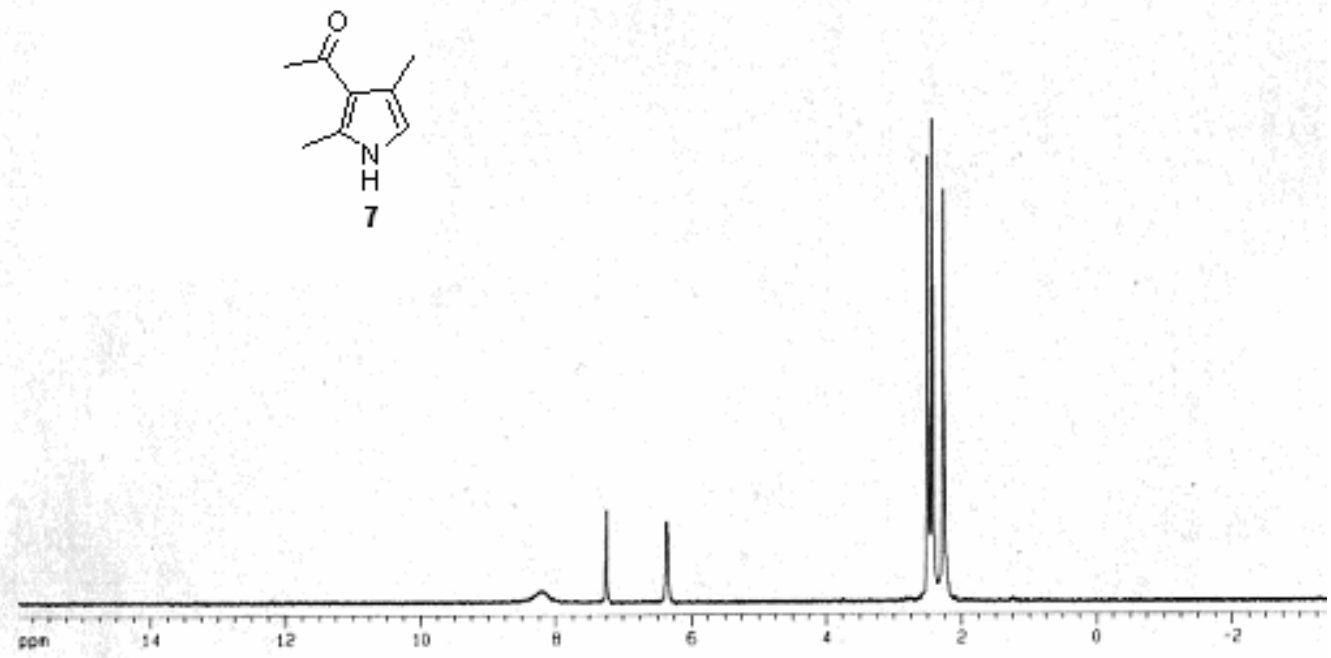




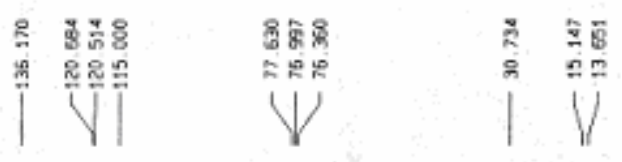<smiles></smiles>
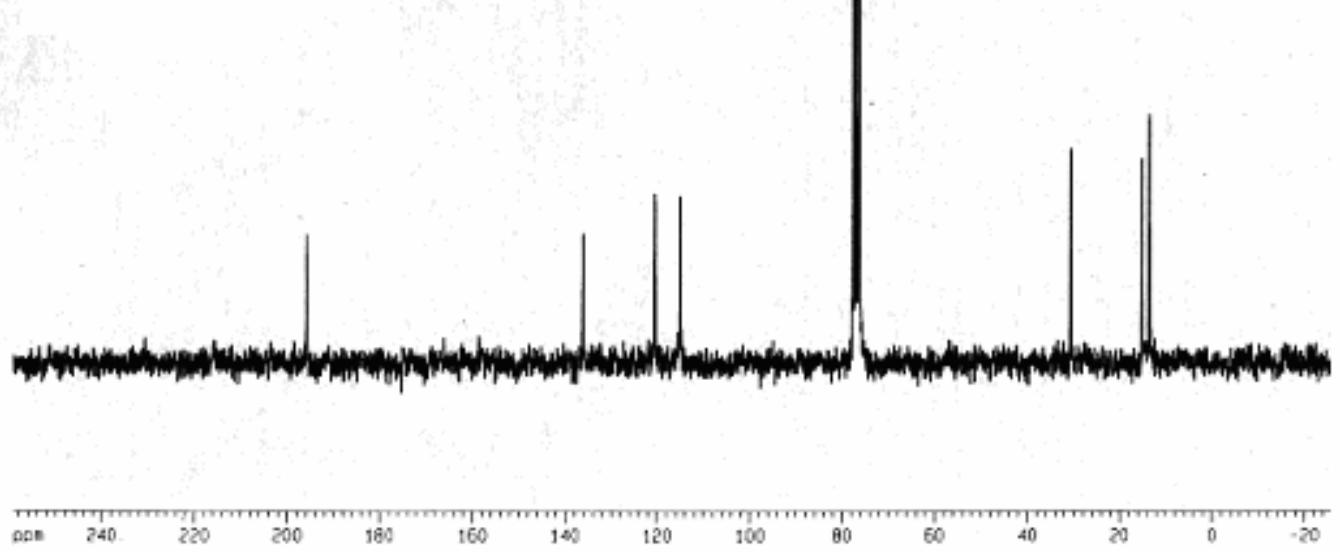


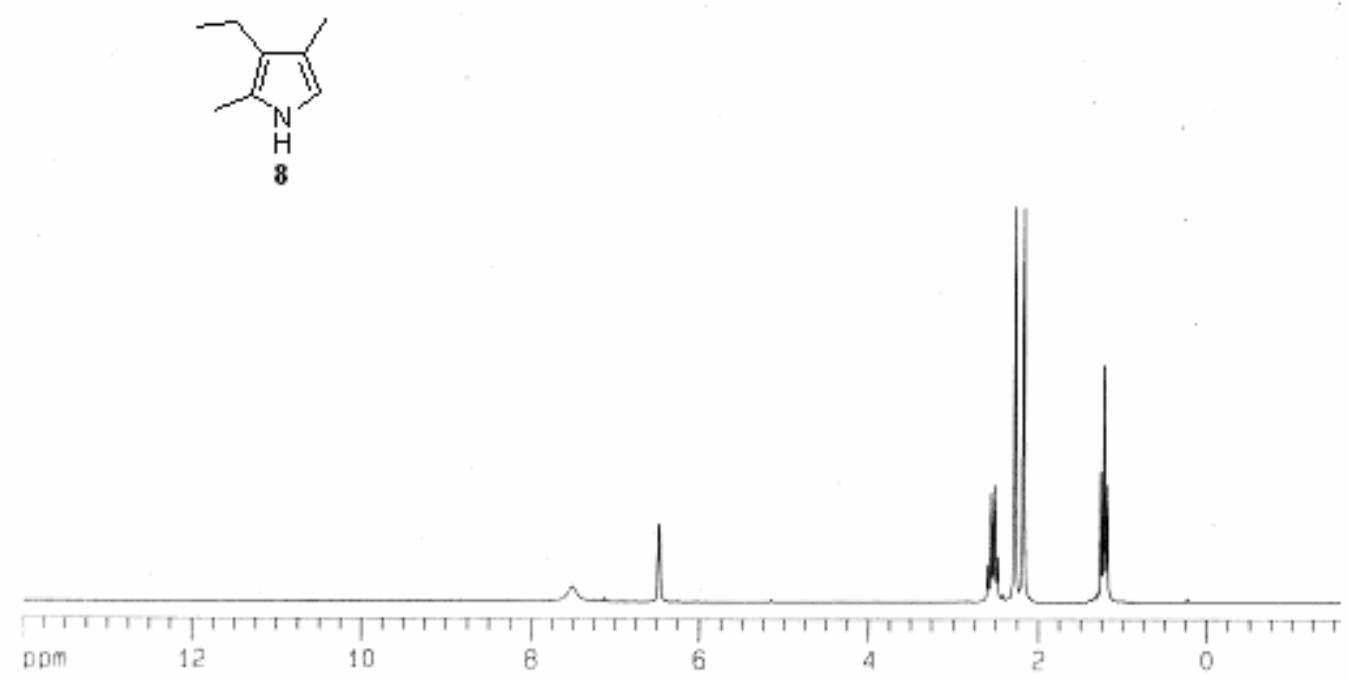




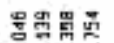

1)

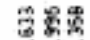

F是

V

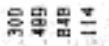

드늠몽

IV
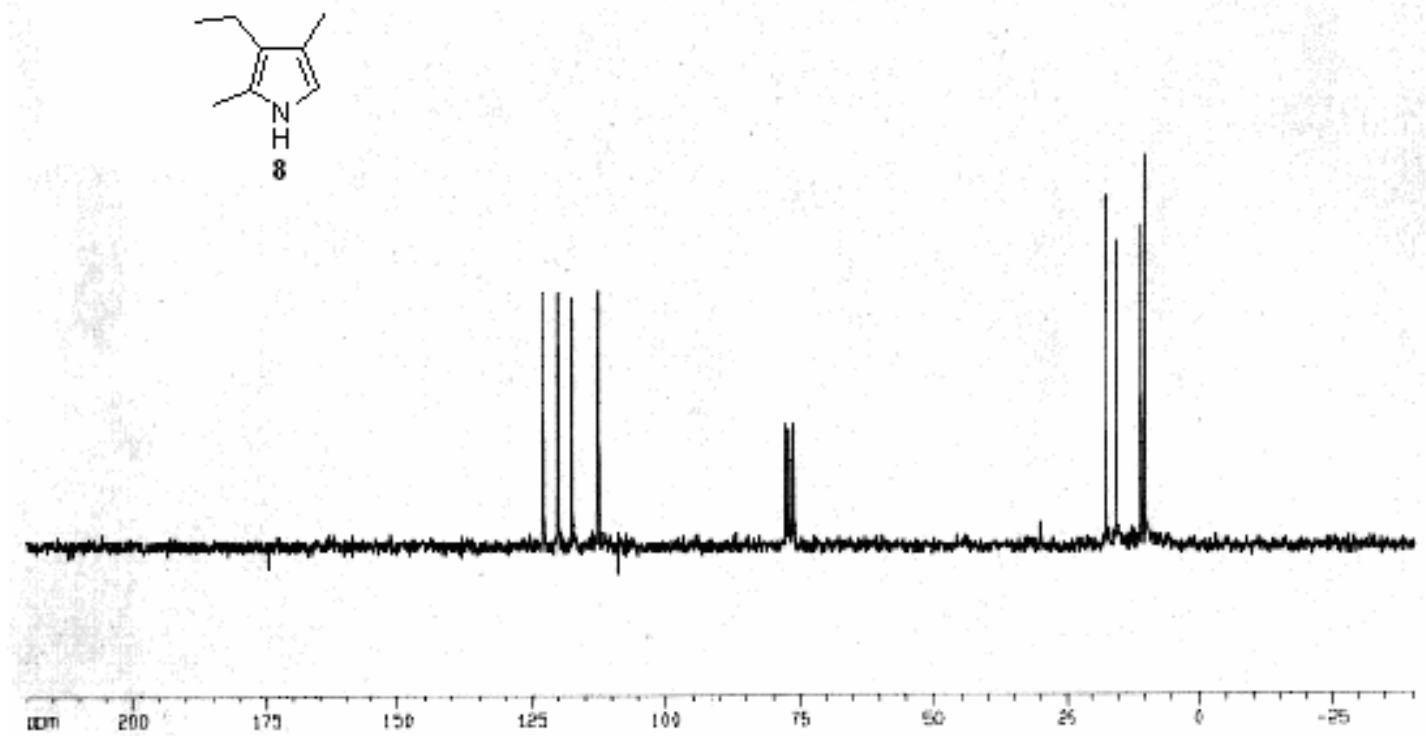


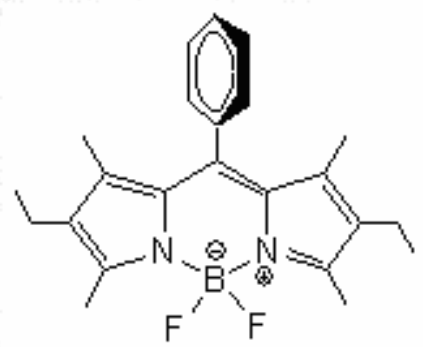

Dye 2

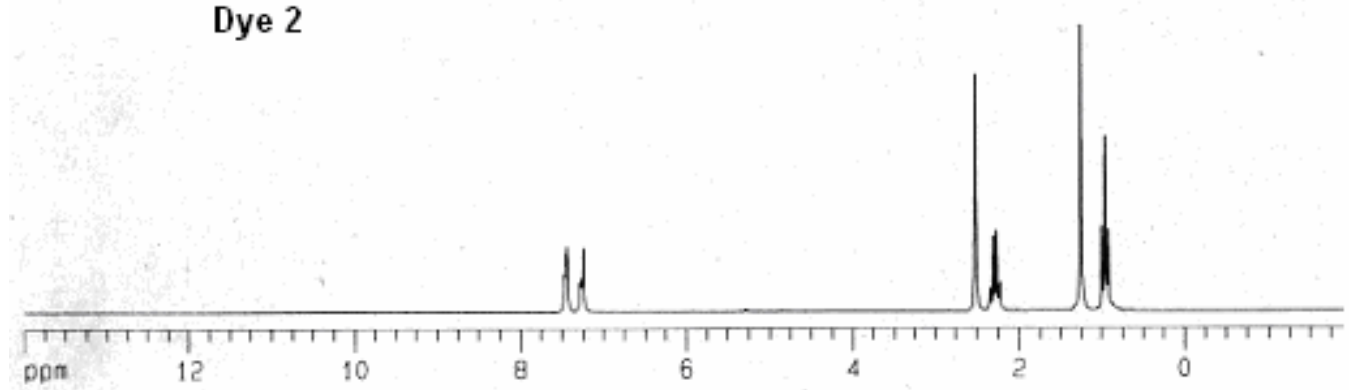




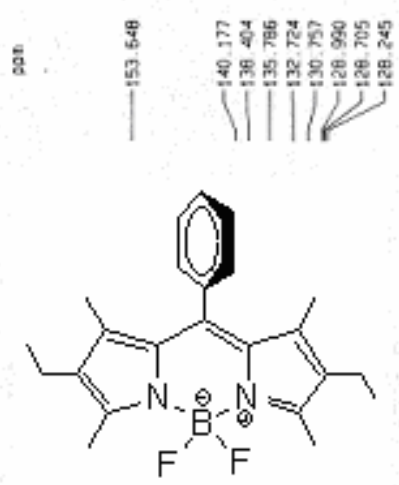

Dye 2
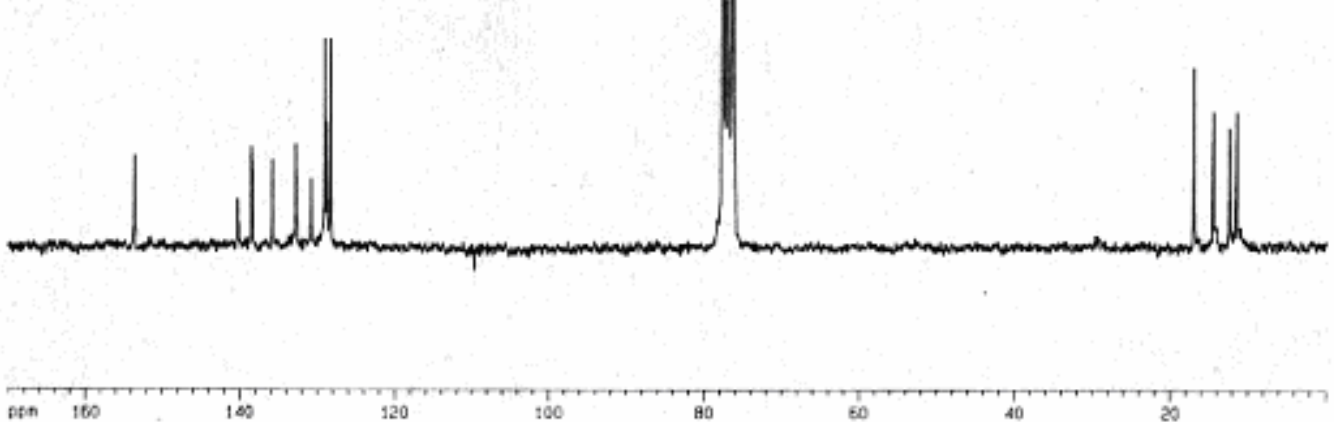

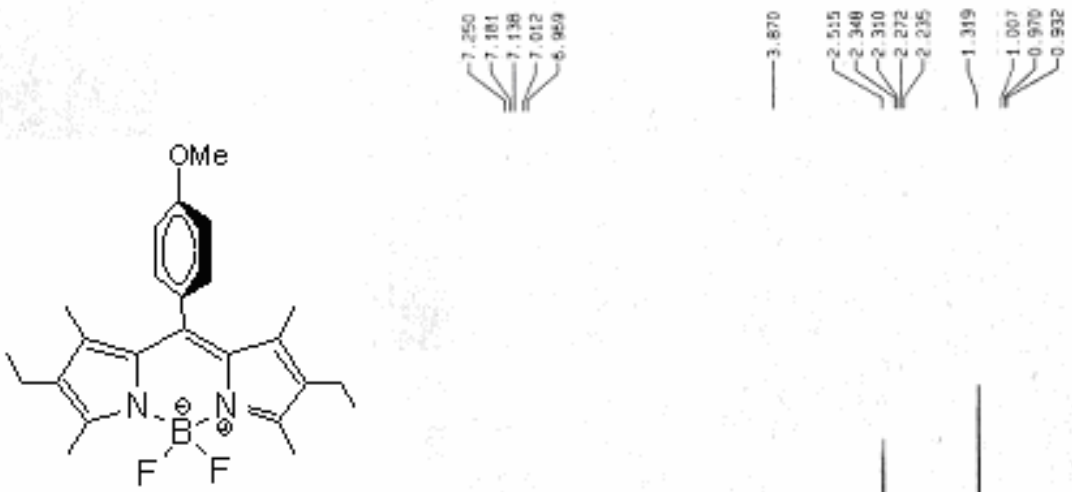

Dye 3

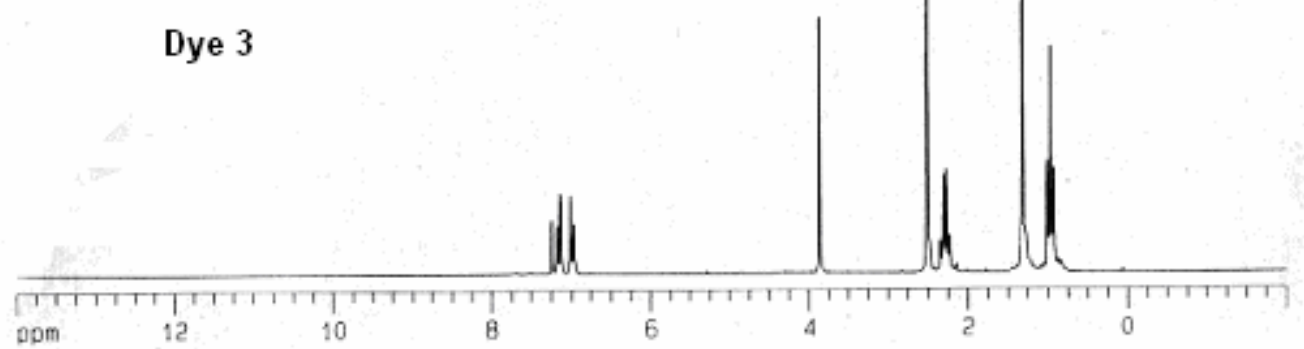


<smiles></smiles>

Dye 3
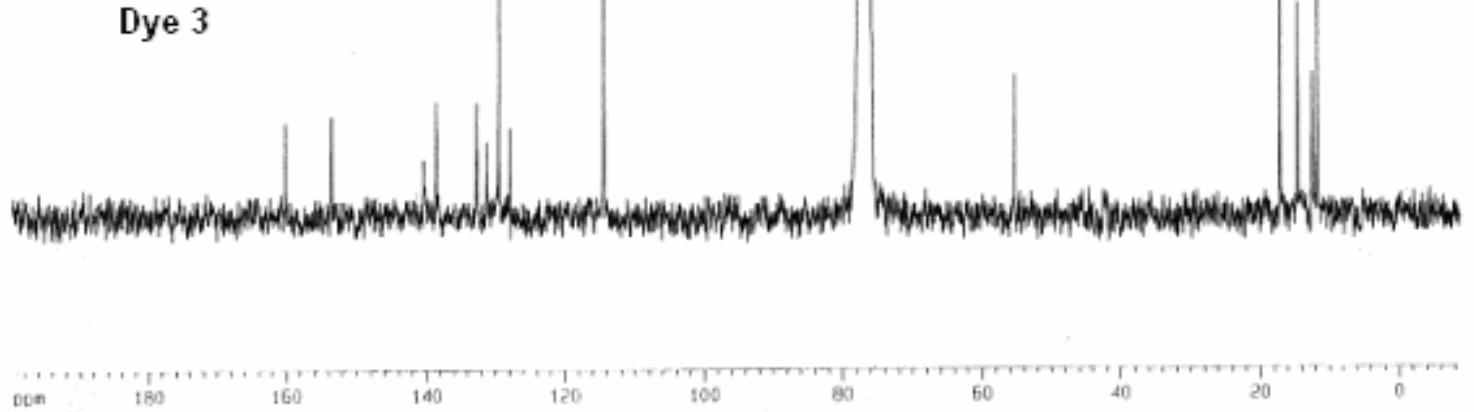

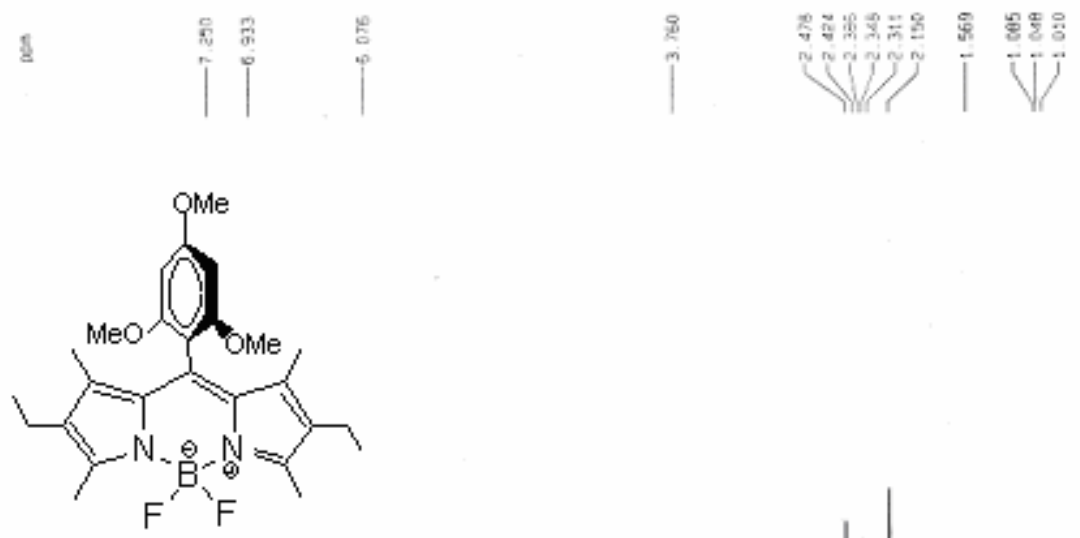

Dye 4

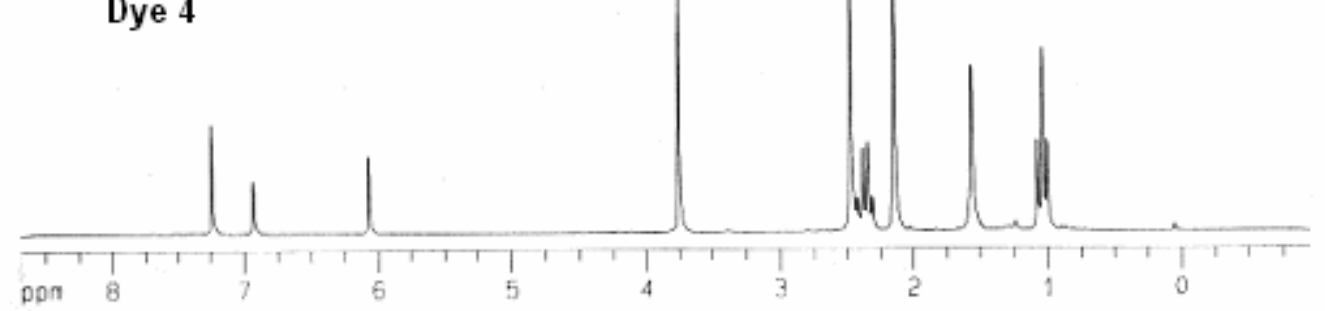



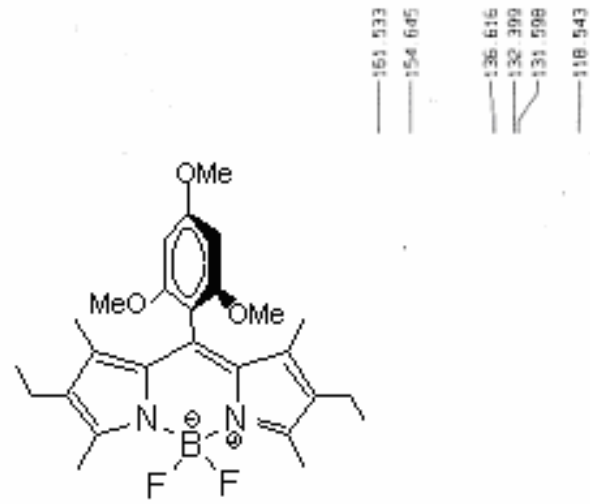

Dye 4
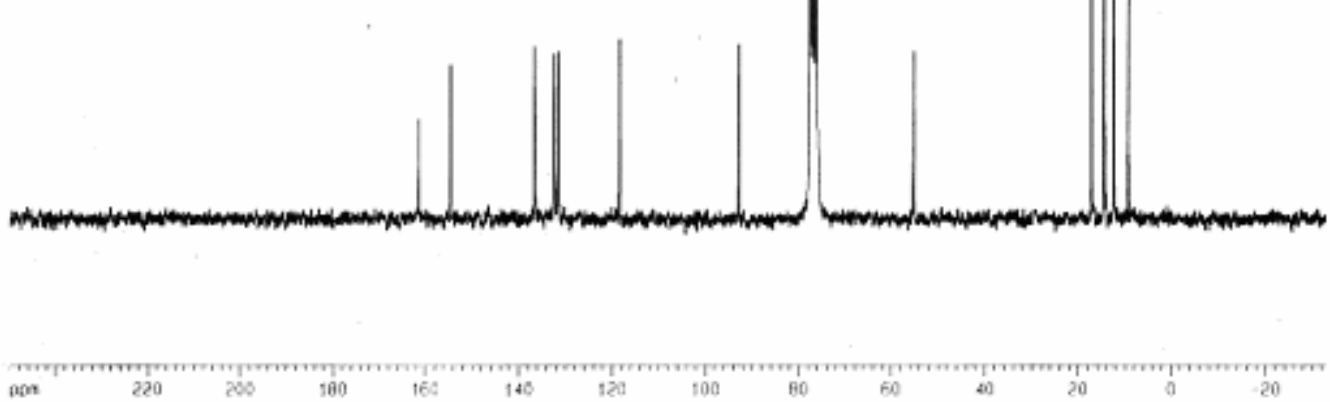
Computational Data. Theoretical calculations have been done at the ab initio Hartree Fock level by using the Programs: SPARTAN'02 and Gaussian-03 + Gview03. In all cases, the ground state geometries of the molecules were optimized by Spartan'02 using a basis set 3-21G*. Final energy calculations were done at single point in the $6-31 \mathrm{G}^{* *}$ basis on the optimized geometry. This was done to achieve economy in calculational efforts. Singlet ground and excited states were done by Spartan Program because of the easy mode of handling data in Spartan. However, we noted difficulties with triplets calculation in our Spartan version for which we made use of Gaussian03 in almost all cases. While comparing Singlet - Triplet difference of energies, we resorted to the same model (Gaussian) of computation for consistency. Relevant information about computational methods, input parameters, Cartesian coordinates of optimized structures along with absolute total energies, state, multiplicity, dipole moments, HOMO LUMO energies etc. are presented in the supporting data of each molecule. Since our work does not concern saddle points/ transition structures determination, imaginary frequency exploration does not arise. 
Dye 1

Geometry optimization done by SPARTAN'02 in 3-21G* basis, finally in $6-31 \mathrm{G}^{* *}$ basis.

$\begin{array}{ll}\text { EXCHANGE } & \text { HF } \\ \text { BASIS } & 6-31 \mathrm{G}^{* *} \\ \text { SYMMETRY } & \text { FALSE } \\ \text { basisprojtype } & 0 \\ \text { BASIS2 } & 3-21 \mathrm{G}(\mathrm{d}) \\ \text { INTSBUFFERSIZE } & 0 \\ \text { VARTHRESH } & 0 \\ \text { SCF_GUESS } & \text { READ } \\ \text { SCF_CONVERGENCE } & 3 \quad \text { \#cheap because redone later } \\ \text { BATCH_JOB_NUMBER } & 0 \quad \text { \#flag for external guess step } \\ \text { EXTERNAL_WVFN } & \text { TRUE } \\ \text { Spin multiplicity } & \text { RHF } \\ \text { NOB } & 119 \\ \text { MERSE_OUTPUT } & \text { TRUE_SPARTAN }\end{array}$


Number of electrons: $\quad 170$

Coordinates (Angstroms)

$\begin{array}{llll}\text { ATOM } & \mathrm{X} & \mathrm{Y} & \mathrm{Z}\end{array}$

\begin{tabular}{|c|c|c|c|}
\hline $1 \mathrm{C}$ & 3.079296 & -2.441759 & -0.239187 \\
\hline $2 \mathrm{C}$ & 2.553506 & -1.031514 & -0.279806 \\
\hline $3 \mathrm{C}$ & 3.360086 & 0.092423 & -0.339844 \\
\hline $4 \mathrm{H}$ & 2.620324 & -3.023521 & 0.550542 \\
\hline $5 \mathrm{~N}$ & 1.246533 & 0.811659 & -0.231144 \\
\hline $6 \mathrm{C}$ & 1.208880 & -0.576727 & -0.221674 \\
\hline $7 \mathrm{H}$ & -2.431620 & 3.172684 & 1.165311 \\
\hline $8 \mathrm{H}$ & 4.144781 & -2.437910 & -0.056744 \\
\hline $9 \mathrm{C}$ & 4.866338 & 0.146223 & -0.399960 \\
\hline $10 \mathrm{H}$ & -3.872914 & 2.861605 & 0.202464 \\
\hline $11 \mathrm{H}$ & 2.915162 & -2.963923 & -1.177616 \\
\hline $12 \mathrm{H}$ & -2.341693 & 3.236293 & -0.578292 \\
\hline $13 \mathrm{C}$ & -2.456344 & 1.262028 & 0.216811 \\
\hline $14 \mathrm{H}$ & 5.172724 & 0.981562 & -1.022012 \\
\hline $15 \mathrm{H}$ & 5.243356 & -0.737227 & -0.904571 \\
\hline $16 C$ & 5.537995 & 0.271132 & 0.973374 \\
\hline 7 & 6.619327 & 0.307704 & 0.875529 \\
\hline
\end{tabular}




\begin{tabular}{|c|c|c|c|}
\hline $18 \mathrm{H}$ & 5.286165 & -0.571476 & 1.610027 \\
\hline $19 \mathrm{H}$ & 5.217262 & 1.173262 & 1.484733 \\
\hline $20 \mathrm{C}$ & 2.502305 & 1.209396 & -0.310881 \\
\hline $21 \mathrm{C}$ & 2.878842 & 2.658984 & -0.349452 \\
\hline $22 \mathrm{H}$ & 2.271220 & 3.188434 & -1.071191 \\
\hline $23 \mathrm{H}$ & 3.920940 & 2.778344 & -0.611417 \\
\hline $24 \mathrm{H}$ & 2.708607 & 3.116295 & 0.618399 \\
\hline $25 \mathrm{C}$ & -3.083668 & -2.377638 & 0.382674 \\
\hline $26 \mathrm{C}$ & -2.546896 & -0.977566 & 0.251446 \\
\hline $27 \mathrm{C}$ & -3.323108 & 0.164025 & 0.373237 \\
\hline $28 \mathrm{H}$ & -2.496085 & -2.978340 & 1.066034 \\
\hline $29 \mathrm{~N}$ & -1.219973 & 0.838075 & 0.030353 \\
\hline $30 \mathrm{C}$ & -1.211204 & -0.550435 & 0.029596 \\
\hline $31 \mathrm{H}$ & -5.400344 & 0.987400 & -1.342541 \\
\hline $32 \mathrm{H}$ & -3.111244 & -2.891205 & -0.574077 \\
\hline $33 \mathrm{C}$ & -4.809335 & 0.249813 & 0.615872 \\
\hline $34 \mathrm{C}$ & -0.012385 & -1.252901 & -0.137974 \\
\hline $35 \mathrm{H}$ & -4.096692 & -2.355613 & 0.759690 \\
\hline $36 \mathrm{C}$ & -0.039392 & -2.758354 & -0.233716 \\
\hline $37 \mathrm{C}$ & -2.802436 & 2.719632 & 0.252992 \\
\hline $38 \mathrm{H}$ & -5.035556 & 1.172954 & 1.139385 \\
\hline o & -5.117656 & -0.542838 & 1.290585 \\
\hline
\end{tabular}




$\begin{array}{cccc}40 \mathrm{C} & -5.651329 & 0.177995 & -0.664145 \\ 41 \mathrm{H} & -6.711443 & 0.246310 & -0.436686 \\ 42 \mathrm{H} & -5.480984 & -0.755033 & -1.192416 \\ 43 \mathrm{~B} & 0.012726 & 1.751353 & -0.195043 \\ 44 \mathrm{~F} & -0.106177 & 2.433182 & -1.389539 \\ & & & \\ 45 \mathrm{~F} & 0.133244 & 2.647313 & 0.845740 \\ & & & \\ 46 \mathrm{H} & -0.954909 & -3.099253 & -0.685381 \\ 47 \mathrm{H} & 0.042302 & -3.210724 & 0.750858 \\ 48 \mathrm{H} & 0.771467 & -3.122126 & -0.840343\end{array}$

Ground state $\left(\mathrm{S}_{0}\right)$ energy $=-1028.88330135$ A.U.

$\mathrm{E}(\mathrm{HOMO})=-6.8022 \mathrm{eV}$

$\mathrm{E}(\mathrm{LUMO})=1.01708 \mathrm{eV}$

Bandgap $=7.8193 \mathrm{eV}$

Ground state dipole moment $($ Debye $)=4.5544$

Excited singlet state $\left(\mathrm{S}_{1}\right)$ dipole moment (Debye)

$$
\begin{array}{llllll}
X & 0.0559 & Y & -4.5477 & Z & 0.4961
\end{array}
$$

Total $\quad 4.5750$

Triplet state Energy: E (UHF) = -1028.84269936 A.U. (Done by G03) 
Dye 2

Geometry optimization done by SPARTAN'02 in $3-21 \mathrm{G}^{*}$ basis, finally in $6-31 \mathrm{G}^{* *}$ basis.

\begin{tabular}{ll} 
EXCHANGE & HF \\
BASIS & $6-31 G^{* *}$ \\
SYMMETRY & FALSE \\
basisprojtype & 0 \\
BASIS2 & $3-21 \mathrm{G}$ (d) \\
INTSBUFFERSIZE & 0 \\
VARTHRESH & 0 \\
SCF_GUESS & READ \\
SCF_CONVERGENCE & $3 \quad$ \#cheap because redone later \\
BATCH_JOB_NUMBER & $0 \quad \#$ flag for external guess step \\
EXTERNAL_WVFN & TRUE \\
GUI & GUI_SPARTAN \\
TERSE_OUTPUT & TRUE \\
JOB TYPE & Single point \\
METHOD & RHF \\
Number of shells: & 193 \\
Number of basis functions: & 555 \\
Molecular charge & 0 \\
\hline &
\end{tabular}


Spin multiplicity 1

Number of electrons: 202

\begin{tabular}{|c|c|c|c|}
\hline \multicolumn{4}{|c|}{ Coordinates (Angstroms) } \\
\hline ATOM & X & Y & Z \\
\hline $1 \mathrm{C}$ & 3.073743 & -1.770097 & -0.314307 \\
\hline $2 \mathrm{C}$ & 2.553630 & -0.358934 & -0.274243 \\
\hline $3 \mathrm{C}$ & 3.347603 & 0.771213 & -0.367145 \\
\hline $4 \mathrm{H}$ & 2.818976 & -2.316512 & 0.584902 \\
\hline $5 \mathrm{~N}$ & 1.237317 & 1.479025 & -0.142983 \\
\hline $6 \mathrm{C}$ & 1.211409 & 0.089262 & -0.136992 \\
\hline $7 \mathrm{H}$ & -2.449003 & 3.792941 & 1.221996 \\
\hline $8 \mathrm{H}$ & 4.151279 & -1.768687 & -0.40323 \\
\hline $9 \mathrm{C}$ & 4.847046 & 0.836933 & -0.518038 \\
\hline $10 \mathrm{H}$ & -3.911956 & 3.475310 & 0.293785 \\
\hline $11 \mathrm{H}$ & 2.671450 & -2.324196 & -1.153153 \\
\hline $12 \mathrm{H}$ & -2.401795 & 3.869277 & -0.521466 \\
\hline $13 \mathrm{C}$ & -2.478726 & 1.889845 & 0.263817 \\
\hline $14 \mathrm{H}$ & 5.112761 & 1.712616 & -1.101714 \\
\hline $15 \mathrm{H}$ & 5.192551 & -0.009663 & -1.102079 \\
\hline $16 \mathrm{C}$ & 5.603586 & 0.873314 & 0.815924 \\
\hline
\end{tabular}




\begin{tabular}{|c|c|c|c|}
\hline $17 \mathrm{H}$ & 6.676557 & 0.923330 & 0.653205 \\
\hline $18 \mathrm{H}$ & 5.394418 & -0.012396 & 1.407740 \\
\hline $19 \mathrm{H}$ & 5.313601 & 1.736586 & 1.406802 \\
\hline $20 \mathrm{C}$ & 2.486203 & 1.883259 & -0.277718 \\
\hline $21 \mathrm{C}$ & 2.849990 & 3.335827 & -0.321151 \\
\hline $22 \mathrm{H}$ & 2.356712 & 3.820943 & -1.154517 \\
\hline $23 \mathrm{H}$ & 3.918346 & 3.464404 & -0.420511 \\
\hline $24 \mathrm{H}$ & 2.519672 & 3.830938 & 0.583416 \\
\hline $25 \mathrm{C}$ & -3.075333 & -1.761866 & 0.323872 \\
\hline $26 \mathrm{C}$ & -2.551970 & -0.352395 & 0.271526 \\
\hline $27 \mathrm{C}$ & -3.341843 & 0.779992 & 0.368313 \\
\hline $28 \mathrm{H}$ & -2.675402 & -2.308409 & 1.168924 \\
\hline $29 \mathrm{~N}$ & -1.232439 & 1.482338 & 0.119035 \\
\hline $30 \mathrm{C}$ & -1.209759 & 0.092251 & 0.118372 \\
\hline $31 \mathrm{H}$ & -5.330240 & 1.722649 & -1.393307 \\
\hline $32 \mathrm{H}$ & -2.820492 & -2.317819 & -0.569315 \\
\hline $33 \mathrm{C}$ & -4.839016 & 0.850744 & 0.538333 \\
\hline $34 \mathrm{C}$ & -0.000122 & -0.591108 & -0.008937 \\
\hline $35 \mathrm{H}$ & -4.152993 & -1.756941 & 0.411662 \\
\hline $36 \mathrm{C}$ & -0.002065 & -2.088451 & -0.006894 \\
\hline $37 \mathrm{C}$ & -2.839740 & 3.342997 & 0.316190 \\
\hline $38 \mathrm{H}$ & -5.094538 & 1.735525 & 1.112763 \\
\hline
\end{tabular}




\begin{tabular}{|c|c|c|c|}
\hline $39 \mathrm{H}$ & -5.177696 & 0.013321 & 1.139465 \\
\hline $40 \mathrm{C}$ & -5.613557 & 0.868870 & -0.785649 \\
\hline $41 \mathrm{H}$ & -6.684157 & 0.923008 & -0.609266 \\
\hline $42 \mathrm{H}$ & -5.413671 & -0.025616 & -1.367319 \\
\hline $43 \mathrm{~B}$ & 0.001895 & 2.410148 & -0.029313 \\
\hline $44 \mathrm{~F}$ & -0.115798 & 3.182597 & -1.167702 \\
\hline $45 \mathrm{~F}$ & 0.117052 & 3.224374 & 1.078425 \\
\hline $46 \mathrm{C}$ & -0.005587 & -4.868490 & -0.004150 \\
\hline $47 \mathrm{C}$ & 0.107381 & -2.787298 & 1.187228 \\
\hline $48 \mathrm{C}$ & -0.113316 & -2.789400 & -1.199696 \\
\hline $49 \mathrm{C}$ & -0.115385 & -4.174516 & -1.197861 \\
\hline $50 \mathrm{C}$ & 0.105912 & -4.172431 & 1.188124 \\
\hline $51 \mathrm{H}$ & 0.193784 & -2.247763 & 2.114039 \\
\hline $52 \mathrm{H}$ & -0.198350 & -2.251355 & -2.127486 \\
\hline $53 \mathrm{H}$ & -0.202422 & -4.709579 & -2.126993 \\
\hline $54 \mathrm{H}$ & 0.191489 & -4.705884 & 2.118310 \\
\hline $55 \mathrm{H}$ & -0.006992 & -5.944031 & -0.003031 \\
\hline
\end{tabular}

Ground state energy E $(\mathrm{RHF})=-1219.40021171$ A. U.

$\mathrm{E}(\mathrm{HOMO})=-6.7374 \mathrm{eV}$

$\mathrm{E}(\mathrm{LUMO})=1.0005 \mathrm{eV}$

Bandgap $=7.7379 \mathrm{eV}$ 
Ground state dipole moment $($ Debye $)=4.8522$

Excited Singlet state $\left(\mathrm{S}_{1}\right)$ Dipole moment (Debye)

$\begin{array}{llllll}X & -0.0182 & Y & -4.9265 & Z & 0.0990\end{array}$

Total 4.9276

UHF triplet state energy: E (UHF) $=-1219.36416778$ A.U. (Done by G03) 
Dye 3

Geometry optimization done by SPARTAN'02 in $3-21 \mathrm{G}^{*}$ basis, finally in $6-31 \mathrm{G}^{* *}$ basis.

\begin{tabular}{ll} 
EXCHANGE & HF \\
BASIS & $6-31 G^{* *}$ \\
SYMMETRY & FALSE \\
basisprojtype & 0 \\
BASIS2 & $3-21 \mathrm{G}(\mathrm{d})$ \\
INTSBUFFERSIZE & 0 \\
VARTHRESH & 0 \\
SCF_GUESS & READ \\
SCF_CONVERGENCE 3 & \#cheap because redone later \\
BATCH_JOB_NUMBER 0 & \#flag for external guess step \\
EXTERNAL_WVFN & TRUE \\
GUI & GUI_SPARTAN \\
TERSE_OUTPUT & TRUE \\
JOB TYPE & Single point \\
METHOD & RHF \\
Number of shells: & 207 \\
Number of basis functions: & 595 \\
Molecular charge & 0 \\
\hline &
\end{tabular}


Spin multiplicity

Number of electrons: $\quad 218$

Coordinates (Angstroms)

$\begin{array}{llll}\text { ATOM } & \mathrm{X} & \mathrm{Y} & \mathrm{Z}\end{array}$

$\begin{array}{llll}1 \mathrm{C} & 2.954288 & -1.517257 & -0.184144\end{array}$

2 C $\quad 2.555999 \quad-0.065978 \quad-0.222656$

$\begin{array}{llll}3 \mathrm{C} & 3.432065 & 0.995649 & -0.392189\end{array}$

$\begin{array}{llll}4 \mathrm{H} & 2.669832 & -1.975684 & 0.753701\end{array}$

$\begin{array}{llll}5 \mathrm{~N} & 1.383712 & 1.871882 & -0.188967\end{array}$

$\begin{array}{llll}6 \mathrm{C} & 1.249264 & 0.479838 & -0.095278\end{array}$

$\begin{array}{llll}7 \mathrm{H} & -1.930458 & 4.550742 & 0.947869\end{array}$

$8 \mathrm{H} \quad 4.024797 \quad-1.613712 \quad-0.296591$

$\begin{array}{llll}9 \mathrm{C} & 4.929058 & 0.934728 & -0.544809\end{array}$

$\begin{array}{llll}10 \mathrm{H} & -3.551529 & 4.316077 & 0.279052\end{array}$

$11 \mathrm{H} \quad 2.475812 \quad-2.075722 \quad-0.977885$

$12 \mathrm{H} \quad-2.142501 \quad 4.452636 \quad-0.780017$

$\begin{array}{llll}13 \mathrm{C} & -2.281431 & 2.592135 & 0.221639\end{array}$

$\begin{array}{llll}14 \mathrm{H} & 5.267714 & 1.761915 & -1.158044\end{array}$

$15 \mathrm{H} \quad 5.211548 \quad 0.029810 \quad-1.069281$

$\begin{array}{llll}16 \mathrm{C} & 5.652414 & 0.983754 & 0.822117\end{array}$ 


\begin{tabular}{|c|c|c|c|}
\hline $17 \mathrm{H}$ & 6.729219 & 0.942832 & 0.693762 \\
\hline $18 \mathrm{H}$ & 5.346654 & 0.147457 & 1.439811 \\
\hline $19 \mathrm{H}$ & 5.402826 & 1.898218 & 1.347424 \\
\hline $20 \mathrm{C}$ & 2.664726 & 2.178163 & -0.362294 \\
\hline $21 \mathrm{C}$ & 3.111225 & 3.600997 & -0.494585 \\
\hline $22 \mathrm{H}$ & 2.603088 & 4.059056 & -1.334149 \\
\hline $23 \mathrm{H}$ & 4.179643 & 3.668217 & -0.632287 \\
\hline $24 \mathrm{H}$ & 2.822437 & 4.150035 & 0.393183 \\
\hline $25 \mathrm{C}$ & -3.125227 & -1.009728 & 0.514834 \\
\hline $26 \mathrm{C}$ & -2.512536 & 0.357942 & 0.371078 \\
\hline $27 \mathrm{C}$ & -3.217239 & 1.552760 & 0.395983 \\
\hline $28 \mathrm{H}$ & -2.706167 & -1.541683 & 1.358400 \\
\hline $29 \mathrm{~N}$ & -1.062650 & 2.076704 & 0.100377 \\
\hline $30 \mathrm{C}$ & -1.140622 & 0.680051 & 0.185963 \\
\hline $31 \mathrm{H}$ & -5.051468 & 2.506544 & -1.462356 \\
\hline $32 \mathrm{H}$ & -2.949183 & -1.609680 & -0.368535 \\
\hline $33 \mathrm{C}$ & -4.704460 & 1.737832 & 0.543199 \\
\hline $34 \mathrm{C}$ & 0.002833 & -0.098659 & 0.088351 \\
\hline $35 \mathrm{H}$ & -4.192929 & -0.927159 & 0.660118 \\
\hline $36 \mathrm{C}$ & -0.110443 & -1.583560 & 0.183961 \\
\hline $37 \mathrm{C}$ & -2.506386 & 4.071224 & 0.166052 \\
\hline $38 \mathrm{H}$ & -4.907905 & 2.679339 & 1.040303 \\
\hline
\end{tabular}




\begin{tabular}{|c|c|c|c|}
\hline $39 \mathrm{H}$ & -5.113406 & 0.961940 & 1.179217 \\
\hline $40 \mathrm{C}$ & -5.428728 & 1.716199 & -0.823954 \\
\hline $41 \mathrm{H}$ & -6.497907 & 1.854332 & -0.699628 \\
\hline $42 \mathrm{H}$ & -5.259163 & 0.770093 & -1.324584 \\
\hline $43 \mathrm{~B}$ & 0.230690 & 2.900465 & -0.104877 \\
\hline $44 \mathrm{~F}$ & 0.154053 & 3.655320 & -1.286393 \\
\hline $45 \mathrm{~F}$ & 0.432175 & 3.782768 & 0.968316 \\
\hline $46 \mathrm{C}$ & -0.322421 & -4.350213 & 0.365941 \\
\hline $47 \mathrm{C}$ & -0.021968 & -2.215452 & 1.418916 \\
\hline $48 \mathrm{C}$ & -0.304363 & -2.345509 & -0.948967 \\
\hline $49 \mathrm{C}$ & -0.410864 & -3.725870 & -0.863926 \\
\hline $50 \mathrm{C}$ & -0.126680 & -3.583612 & 1.509111 \\
\hline $51 \mathrm{H}$ & 0.128421 & -1.629447 & 2.304087 \\
\hline $52 \mathrm{H}$ & -0.373542 & -1.865873 & -1.905598 \\
\hline $53 \mathrm{H}$ & -0.561071 & -4.292301 & -1.758526 \\
\hline $54 \mathrm{H}$ & -0.061399 & -4.088948 & 2.449457 \\
\hline $55 \mathrm{O}$ & -0.413951 & -5.700781 & 0.558091 \\
\hline $56 \mathrm{C}$ & -0.618392 & -6.595472 & -0.548856 \\
\hline $57 \mathrm{H}$ & -0.651407 & -7.582137 & -0.117703 \\
\hline $58 \mathrm{H}$ & 0.197115 & -6.535494 & -1.258781 \\
\hline $59 \mathrm{H}$ & -1.553211 & -6.387722 & -1.054454 \\
\hline
\end{tabular}


Ground state Energy E (RHF) $=-1333.28088029$ A.U.

$\mathrm{E}-\mathrm{HOMO}=-6.7158 \mathrm{eV}$

E-LUMO $=0.9988 \mathrm{eV}$

Bandgap $=7.7145 \mathrm{eV}$

Dipole Moment $($ Debye $)=5.8935$

Excited singlet state $\left(\mathrm{S}_{1}\right)$ Dipole moment (Debye)

$\begin{array}{llllll}\mathrm{X} & -0.5851 & \mathrm{Y} & -5.8409 & \mathrm{Z} & -1.0357\end{array}$

Total $\quad 5.9608$

UHF Triplet state energy $=-13333.24497394$ A. U. (Done by G03) 
Dye 4

Geometry optimization done by SPARTAN'02 in 3-21G* basis, finally in $6-31 \mathrm{G}^{* *}$ basis.

\begin{tabular}{|c|c|}
\hline EXCHANGE & $\mathrm{HF}$ \\
\hline BASIS & $6-31 G^{* *}$ \\
\hline SYMMETRY & FALSE \\
\hline basisprojtype & 0 \\
\hline BASIS2 & $3-21 G(d)$ \\
\hline INTSBUFFERSIZE & 0 \\
\hline VARTHRESH & 0 \\
\hline SCF_GUESS & READ \\
\hline SCF_CONVERGENCE 3 & \#cheap because redone later \\
\hline BATCH_JOB_NUMBER 0 & \#flag for external guess step \\
\hline EXTERNAL_WVFN & TRUE \\
\hline GUI & GUI_SPARTAN \\
\hline TERSE_OUTPUT & TRUE \\
\hline JOB TYPE & Single point \\
\hline METHOD & RHF \\
\hline Number of shells: & 235 \\
\hline Number of basis functions: & 675 \\
\hline Molecular charge & 0 \\
\hline
\end{tabular}


Spin multiplicity 1

Number of electrons: $\quad 250$

\begin{tabular}{|c|c|c|c|}
\hline \multicolumn{4}{|c|}{ Coordinates (Angstroms) } \\
\hline ATOM & $\mathrm{X}$ & $\mathrm{Y}$ & Z \\
\hline $1 \mathrm{C}$ & 3.209297 & -0.389695 & -0.276616 \\
\hline $2 \mathrm{C}$ & 2.352145 & 0.847045 & -0.265154 \\
\hline $3 \mathrm{C}$ & 2.832420 & 2.139441 & -0.438105 \\
\hline $4 \mathrm{H}$ & 2.969066 & -1.054348 & 0.541549 \\
\hline $5 \mathrm{~N}$ & 0.613624 & 2.294350 & -0.214913 \\
\hline $6 \mathrm{C}$ & 0.942297 & 0.934836 & -0.122259 \\
\hline $7 \mathrm{H}$ & -3.372284 & 3.763306 & 0.942840 \\
\hline $8 \mathrm{H}$ & 4.253403 & -0.121084 & -0.198677 \\
\hline $9 \mathrm{C}$ & 4.266575 & 2.565551 & -0.611693 \\
\hline $10 \mathrm{H}$ & -4.835452 & 3.009197 & 0.293897 \\
\hline $11 \mathrm{H}$ & 3.057596 & -0.927353 & -1.203678 \\
\hline $12 \mathrm{H}$ & -3.555955 & 3.585673 & -0.781624 \\
\hline $13 \mathrm{C}$ & -3.075614 & 1.790102 & 0.233349 \\
\hline $14 \mathrm{H}$ & 4.310797 & 3.455349 & -1.229288 \\
\hline $15 \mathrm{H}$ & 4.818634 & 1.798391 & -1.141110 \\
\hline $16 \mathrm{C}$ & 4.954497 & 2.853060 & 0.744176 \\
\hline
\end{tabular}




\begin{tabular}{|c|c|c|c|}
\hline $17 \mathrm{H}$ & 5.984473 & 3.163025 & 0.599971 \\
\hline $18 \mathrm{H}$ & 4.945959 & 1.965900 & 1.366736 \\
\hline $19 \mathrm{H}$ & 4.429203 & 3.639746 & 1.273125 \\
\hline $20 \mathrm{C}$ & 1.725004 & 3.003971 & -0.398914 \\
\hline $21 \mathrm{C}$ & 1.679342 & 4.494424 & -0.534353 \\
\hline $22 \mathrm{H}$ & 1.046883 & 4.759449 & -1.372757 \\
\hline $23 \mathrm{H}$ & 2.666432 & 4.907334 & -0.677274 \\
\hline $24 \mathrm{H}$ & 1.230139 & 4.921533 & 0.353514 \\
\hline $25 \mathrm{C}$ & -2.708985 & -1.879956 & 0.602777 \\
\hline $26 \mathrm{C}$ & -2.572426 & -0.396778 & 0.398525 \\
\hline $27 \mathrm{C}$ & -3.623865 & 0.506153 & 0.424129 \\
\hline $28 \mathrm{H}$ & -2.288919 & -2.150476 & 1.562509 \\
\hline $29 \mathrm{~N}$ & -1.756690 & 1.695425 & 0.101526 \\
\hline $30 \mathrm{C}$ & -1.377103 & 0.347489 & 0.194548 \\
\hline $31 \mathrm{H}$ & -5.700743 & 0.811278 & -1.399313 \\
\hline $32 \mathrm{H}$ & -2.189380 & -2.439551 & -0.163308 \\
\hline $33 \mathrm{C}$ & -5.088635 & 0.202439 & 0.598184 \\
\hline $34 \mathrm{C}$ & -0.047960 & -0.025018 & 0.075111 \\
\hline $35 \mathrm{H}$ & -3.750636 & -2.167634 & 0.584345 \\
\hline $36 \mathrm{C}$ & 0.334684 & -1.462929 & 0.124151 \\
\hline $37 \mathrm{C}$ & -3.768442 & 3.115622 & 0.170660 \\
\hline $38 \mathrm{H}$ & -5.575922 & 1.029620 & 1.101592 \\
\hline
\end{tabular}




\begin{tabular}{|c|c|c|c|}
\hline $39 \mathrm{H}$ & -5.212619 & -0.661437 & 1.239763 \\
\hline $40 \mathrm{C}$ & -5.792459 & -0.055745 & -0.755404 \\
\hline $41 \mathrm{H}$ & -6.846979 & -0.267491 & -0.611248 \\
\hline $42 \mathrm{H}$ & -5.337884 & -0.899785 & -1.261013 \\
\hline $43 \mathrm{~B}$ & -0.806746 & 2.892455 & -0.125856 \\
\hline $44 \mathrm{~F}$ & -1.136847 & 3.564150 & -1.315512 \\
\hline $45 \mathrm{~F}$ & -0.897512 & 3.807646 & 0.934973 \\
\hline $46 \mathrm{C}$ & 1.128611 & -4.130267 & 0.286837 \\
\hline $47 \mathrm{C}$ & 0.327965 & -2.142242 & 1.341285 \\
\hline $48 \mathrm{C}$ & 0.737893 & -2.149261 & -1.007291 \\
\hline $49 \mathrm{C}$ & 1.124533 & -3.478738 & -0.926661 \\
\hline $50 \mathrm{C}$ & 0.726443 & -3.450952 & 1.428370 \\
\hline 510 & -0.132118 & -1.508124 & 2.476310 \\
\hline $52 \mathrm{O}$ & 0.862149 & -1.574112 & -2.247282 \\
\hline $53 \mathrm{H}$ & 1.426829 & -3.953901 & -1.832775 \\
\hline $54 \mathrm{H}$ & 0.707144 & -3.963665 & 2.364750 \\
\hline $55 \mathrm{O}$ & 1.500351 & -5.429212 & 0.459233 \\
\hline $56 \mathrm{C}$ & 1.932539 & -6.231568 & -0.655004 \\
\hline $57 \mathrm{H}$ & 2.162663 & -7.198091 & -0.238869 \\
\hline $58 \mathrm{H}$ & 2.816371 & -5.812795 & -1.118874 \\
\hline $59 \mathrm{H}$ & 1.147778 & -6.328915 & -1.394218 \\
\hline
\end{tabular}




$\begin{array}{llll}60 \mathrm{C} & -0.244682 & -0.878335 & -2.891989 \\ 61 \mathrm{C} & 0.831514 & -0.778489 & 3.292875 \\ 62 \mathrm{H} & -1.175782 & -1.401461 & -2.721608 \\ 63 \mathrm{H} & -0.004741 & -0.884466 & -3.942563 \\ 64 \mathrm{H} & -0.320038 & 0.133961 & -2.535035 \\ 65 \mathrm{H} & 1.606986 & -1.445978 & 3.644591 \\ 66 \mathrm{H} & 0.267406 & -0.391057 & 4.124336 \\ 67 \mathrm{H} & 1.267773 & 0.032987 & 2.729701\end{array}$

Ground state $\left(\mathrm{S}_{0}\right)$ energy: $\mathrm{E}(\mathrm{RHF})=-1561.04490043$ A.U.

$\mathrm{E}(\mathrm{HOMO})=-6.7123 \mathrm{eV}$

$\mathrm{E}(\mathrm{LUMO})=0.9468 \mathrm{eV}$

Bandgap $=7.6590 \mathrm{eV}$

Ground state dipole moment $($ Debye $)=5.0115$

Excited Singlet state $\left(\mathrm{S}_{1}\right)$ Dipole Moments (Debye)

$$
\begin{array}{llllll}
X & 1.5354 & Y & -4.7204 & Z & -0.7884
\end{array}
$$

Total $\quad 5.0261$

UHF-Triplet state Energy $=-1561.00958182$ A. U. (Done by G03) 
11

Geometry optimization done by SPARTAN'02 in 3-21G* basis, finally in $6-31 \mathrm{G}^{* *}$ basis.

\begin{tabular}{ll} 
EXCHANGE & HF \\
BASIS & $6-31 \mathrm{G}^{* *}$ \\
SYMMETRY & FALSE \\
basisprojtype & 0 \\
BASIS2 & $3-21 \mathrm{G}(\mathrm{d})$ \\
INTSBUFFERSIZE & 0 \\
VARTHRESH & 0 \\
SCF_GUESS & READ \\
SCF_CONVERGENCE & $3 \quad$ \#cheap because redone later \\
BATCH_JOB_NUMBER & 0 \\
EXTERNAL_WVFN & TRUE for external guess step \\
GUI & 157 \\
TERSE_OUTPUT & GUI_SPARTAN \\
Number of shells: & TRUE \\
Number of basis functions: & 162 \\
\hline
\end{tabular}


Coordinates (Angstroms)

$\begin{array}{llll}\text { ATOM } & \mathrm{X} & \mathrm{Y} & \mathrm{Z}\end{array}$

\begin{tabular}{|c|c|c|c|}
\hline $1 \mathrm{C}$ & 2.937016 & -2.651989 & -0.320886 \\
\hline $2 \mathrm{C}$ & 2.518133 & -1.211695 & -0.268215 \\
\hline $3 \mathrm{C}$ & 3.344654 & -0.104549 & -0.353650 \\
\hline $4 \mathrm{H}$ & 2.090856 & -3.322617 & -0.251893 \\
\hline $5 \mathrm{~N}$ & 1.244494 & 0.655898 & -0.133517 \\
\hline $6 \mathrm{C}$ & 1.202236 & -0.728526 & -0.129251 \\
\hline $7 \mathrm{H}$ & -2.656102 & 2.956441 & -0.627185 \\
\hline $8 \mathrm{H}$ & 3.614768 & -2.891462 & 0.492475 \\
\hline $9 \mathrm{C}$ & 4.845233 & -0.099139 & -0.493760 \\
\hline $10 \mathrm{H}$ & -2.358366 & 2.987646 & 1.092700 \\
\hline $11 \mathrm{H}$ & 3.453410 & -2.870727 & -1.250642 \\
\hline $12 \mathrm{H}$ & -3.963922 & 2.576830 & 0.490648 \\
\hline $13 \mathrm{C}$ & -2.506493 & 1.029787 & 0.267744 \\
\hline $14 \mathrm{H}$ & 5.150297 & 0.700428 & -1.163174 \\
\hline $15 \mathrm{H}$ & 5.158618 & -1.020023 & -0.977320 \\
\hline $16 \mathrm{C}$ & 5.592181 & 0.044749 & 0.838505 \\
\hline $17 \mathrm{H}$ & 6.667283 & 0.035959 & 0.683365 \\
\hline $18 \mathrm{H}$ & 5.343580 & -0.767309 & 1.515005 \\
\hline
\end{tabular}




\begin{tabular}{|c|c|c|c|}
\hline $19 \mathrm{H}$ & 5.333028 & 0.973985 & 1.335947 \\
\hline $20 \mathrm{C}$ & 2.507194 & 1.028841 & -0.265546 \\
\hline $21 \mathrm{C}$ & 2.904567 & 2.471709 & -0.308255 \\
\hline $22 \mathrm{H}$ & 2.636665 & 2.960072 & 0.621403 \\
\hline $23 \mathrm{H}$ & 2.377371 & 2.981498 & -1.105069 \\
\hline $24 \mathrm{H}$ & 3.969120 & 2.575424 & -0.465255 \\
\hline $25 \mathrm{C}$ & -2.937937 & -2.650781 & 0.315469 \\
\hline $26 \mathrm{C}$ & -2.518246 & -1.210813 & 0.264702 \\
\hline $27 \mathrm{C}$ & -3.344169 & -0.103284 & 0.354672 \\
\hline $28 \mathrm{H}$ & -3.438071 & -2.874617 & 1.252695 \\
\hline $29 \mathrm{~N}$ & -1.243839 & 0.656384 & 0.133929 \\
\hline $30 \mathrm{C}$ & -1.202549 & -0.728030 & 0.125490 \\
\hline $31 \mathrm{H}$ & -5.337590 & 0.965882 & -1.335886 \\
\hline $32 \mathrm{H}$ & -2.094018 & -3.323061 & 0.226145 \\
\hline $33 \mathrm{C}$ & -4.844548 & -0.098808 & 0.497171 \\
\hline $34 \mathrm{C}$ & -0.000183 & -1.394698 & -0.002662 \\
\hline $35 \mathrm{H}$ & -3.630147 & -2.883576 & -0.487229 \\
\hline $36 \mathrm{H}$ & -0.000437 & -2.467997 & -0.004045 \\
\hline $37 \mathrm{C}$ & -2.902847 & 2.472914 & 0.310859 \\
\hline $38 \mathrm{H}$ & -5.149393 & 0.703714 & 1.162738 \\
\hline $39 \mathrm{H}$ & -5.155869 & -1.017387 & 0.986002 \\
\hline $40 \mathrm{C}$ & -5.593879 & 0.037926 & -0.834574 \\
\hline
\end{tabular}




$\begin{array}{llll}41 \mathrm{H} & -6.668774 & 0.027788 & -0.677789 \\ 42 \mathrm{H} & -5.344856 & -0.776388 & -1.507884 \\ 43 \mathrm{~B} & 0.000220 & 1.591795 & -0.000926 \\ & & & \\ 44 \mathrm{~F} & -0.122302 & 2.379963 & -1.123784 \\ 45 \mathrm{~F} & 0.123613 & 2.381843 & 1.120731\end{array}$

SCF Energy is -989.854903150 A. U.

$\mathrm{E}(\mathrm{HOMO})=-6.80924 \mathrm{eV}$

$\mathrm{E}(\mathrm{LUMO})=0.91720 \mathrm{eV}$

Bandgap $=7.72645 \mathrm{eV}$

Dipole Moment $($ Debye $)=4.0618$

Excited singlet state $\left(\mathrm{S}_{1}\right)$ dipole moment (Debye)

$\begin{array}{llllll}\mathrm{X} & 0.0126 & \mathrm{Y} & -4.0875 & \mathrm{Z} & -0.0001\end{array}$

Total 4.0875 
13

Geometry optimization done by SPARTAN'02 in 6-31G** basis.

$\begin{array}{ll}\text { JOBTYPE } & \text { OPT } \\ \text { TIDY_SYM } & \text { TRUE } \\ \text { EXCHANGE } & \text { HF } \\ \text { BASIS } & 6-31 G^{* *} \\ \text { USE_SP_DERIV } & 2 \\ \text { GEOM_OPT_HESSIAN } & \text { READ } \\ \text { EXTERNAL_HESSIAN } & \text { TRUE } \\ \text { GUI } & \text { GUI_SPARTAN } \\ \text { TERSE_OUTPUT } & \text { TRUE } \\ \text { Run type } & \text { Molecular gradient (no SCF) } \\ \text { MODEL } & \text { RHF/ABASIS } \\ \text { Number of shells: } & 84 \\ \text { Number of basis functions: } & 240 \\ \text { Molecular charge: } & 0 \\ \text { Number of electrons: } & 1 \\ \text { Nin multiplicity: } & 90 \\ \text { N } & \end{array}$


Coordinates (Angstroms)

$\begin{array}{llll}\text { ATOM } & \mathrm{X} & \mathrm{Y} & \mathrm{Z}\end{array}$

\begin{tabular}{|c|c|c|c|}
\hline $1 \mathrm{O}$ & 2.391544 & -1.297917 & 0.000000 \\
\hline $2 \mathrm{C}$ & 1.239460 & -0.604473 & 0.000000 \\
\hline $3 \mathrm{C}$ & -1.249316 & 0.626068 & 0.000000 \\
\hline $4 \mathrm{C}$ & 1.166849 & 0.768906 & 0.000000 \\
\hline $5 \mathrm{C}$ & 0.082467 & -1.394974 & 0.000000 \\
\hline $6 \mathrm{C}$ & -1.143219 & -0.771167 & 0.000000 \\
\hline $7 \mathrm{C}$ & -0.096241 & 1.375640 & 0.000000 \\
\hline $8 \mathrm{H}$ & 2.030955 & 1.401022 & 0.000000 \\
\hline $9 \mathrm{H}$ & 0.197843 & -2.459370 & 0.000000 \\
\hline $10 \mathrm{O}$ & -2.319801 & -1.422179 & 0.000000 \\
\hline $11 \mathrm{O}$ & -0.071743 & 2.720097 & 0.000000 \\
\hline $12 \mathrm{H}$ & -2.228798 & 1.058348 & 0.000000 \\
\hline $13 \mathrm{C}$ & 3.614517 & -0.615197 & 0.000000 \\
\hline $14 \mathrm{C}$ & -1.274483 & 3.437862 & 0.000000 \\
\hline $15 \mathrm{C}$ & -2.340035 & -2.822666 & 0.000000 \\
\hline $16 \mathrm{H}$ & 3.722871 & 0.003241 & 0.885037 \\
\hline $17 \mathrm{H}$ & 3.722871 & 0.003241 & -0.885037 \\
\hline $18 \mathrm{H}$ & 4.383426 & -1.373982 & 0.000000 \\
\hline
\end{tabular}




$\begin{array}{llll}19 \mathrm{H} & -1.864242 & 3.222481 & 0.885037 \\ 20 \mathrm{H} & -1.864242 & 3.222481 & -0.885037 \\ 21 \mathrm{H} & -1.001810 & 4.483150 & 0.000000 \\ 22 \mathrm{H} & -1.858629 & -3.225722 & 0.885037 \\ 23 \mathrm{H} & -1.858629 & -3.225722 & -0.885037 \\ 24 \mathrm{H} & -3.381617 & -3.109167 & 0.000000\end{array}$

Energy $=-572.367920760$ A. U.

$\mathrm{E}(\mathrm{HOMO})=-8.21270 \mathrm{eV}$

$\mathrm{E}(\mathrm{LUMO})=4.75765 \mathrm{eV}$

Bandgap $=12.9703 \mathrm{eV}$

Dipole Moment $($ Debye $)=0.0000$

Excited Singlet state $\left(\mathrm{S}_{1}\right)$ Dipole Moment $($ Debye $)=0.0017$ 